\title{
Joint selection of the MCS's and power allocation coefficients in the two-user downlink PD-NOMA system
}

\author{
Yakov Kryukov*, Dmitriy Pokamestov, and Eugeniy Rogozhnikov \\ Tomsk State University of Control and Radioelectronics, Department of Telecommunications and \\ Basic Principles of Radio Engineering, 634045, Tomsk, Russia
}

\begin{abstract}
Power Domain Non-Orthogonal Multiple Access (PD-NOMA) is a perspective multiplexing technique for future cellular networks. Nevertheless, it is poorly studied and not applied in the existing systems due to the complexity of PD-NOMA signal processing, resource scheduling, and power allocation. The issue is that a modulation and coding scheme (MCS) selection, including power allocation, is a cooperative procedure considering the channel state information of each multiplexed user. It can be solved by enumerating all possible multiplexing combinations but at the expense of the high computational complexity. In our work, we propose a composed table with the joint MCS's, which can be selected by the base station (BS) for the user multiplexing in a downlink PD-NOMA system based on their signal-to-noise (SNR) ratios. It allows selecting two MCS's with two power allocation coefficients for both users and guarantees the $10 \%$ block error rate (BLER) performance in the additive white Gaussian noise (AWGN) channel. The joint MCS selection method is based on a max-rate scheduling strategy and provides system capacity maximization ignoring fairness between users. The proposed table is given in the Appendix.
\end{abstract}

\section{Introduction}

PD-NOMA is one of the perspective techniques for user multiplexing in future networks. It has been shown [1-5] that PD-NOMA allows increasing the system capacity in the multiuser communication systems such as cellular (3GPP long term evolution, LTE) and wireless local area networks (IEEE 802.11). The basic PD-NOMA idea is the nonorthogonal multiplexing of several users in a power domain of the same time-frequency resource segment by the superposition of their signals with different power weights. Thus, there is a controlled co-channel interference that is cancelled at the receiver side by the successive interference cancellation (SIC) method [6]. The issue is that user signals are dependent on each other, and it should be considered during resource scheduling, cooperative MCS selection, and multiplexing.

\footnotetext{
* Corresponding author: kryukov.tusur@gmail.com
} 
To achieve the maximum system capacity, it is necessary to use the MCS that has the highest spectral efficiency (SE) and guarantees the required error rate performance within the given channel conditions. Therefore, adaptive modulation and coding (AMC) has been proposed [7,8]. It is a simple approach that selects the most efficient MCS according to the propagation channel quality. The AMC is widely used in actual wireless networks with orthogonal multiple access (OMA). Thus, in conventional OMA systems, MCS's for different users are independently selected because they are orthogonal to each other. Unlike OMA, in PD-NOMA systems, the users are non-orthogonal in the power domain, and it is a reason for the joint MCS selection. Besides, the power allocation coefficients should be calculated by taking into account the channel quality of each user. It can be carried out by enumerating all possible multiplexing configurations and selecting the most effective of them (joint MCS's and power allocation coefficients) at the expense of the high computational complexity.

Many researchers have developed power allocation schemes for PD-NOMA within the conditions of the Shannon system model, considering the infinite number of MCS levels. However, during the literature searching, we have found the only paper devoted to the joint MCS selection in PD-NOMA systems using finite number of MCS levels from the practical system and considering the efficiency of used error-correcting methods. In [9], the authors propose their method of a joint power allocation and MCS selection considering max-min user fairness and determining the power allocation coefficient according to the MCS level of the user with a poor channel. It allocates the maximum power shared to the user with better channel conditions while guaranteeing the decoding of the second user. However, their algorithm is iterative, so it has high computational complexity.

In our work, we propose a table that makes it possible to select the pair of MCS levels depending on the user SNR values considering the max C/I scheduling strategy. It maximizes the system throughput instantly by selecting two MCS's that have the highest sum SE. This approach has a very low computing complexity and may be a temporary solution for the AMC in a two-user PD-NOMA system until an optimal approach is found.

\section{Downlink PD-NOMA system}

We are using the PD-NOMA system model with multiplexing of two users in the power domain. According to its principles, the group signal is a superposition of two user signals with different power weights. The user with a weak propagation channel gets the largest power share compared to the user with a strong propagation channel. We sorted users in the ascending order of their channel quality and identified the weak user $\left(\mathrm{UE}_{1}\right)$ with power allocation coefficient $p_{1}$ and the strong user $\left(\mathrm{UE}_{2}\right)$ with $p_{2}$; therefore, $p_{1}>p_{2}$ and $p_{1}+p_{2}=1$. The downlink group signal $\mathbf{s}$ at the $\mathrm{BS}$ output is given by

$$
\mathbf{s}=\sqrt{p_{1}} \mathbf{x}+\sqrt{p_{2}} \mathbf{y}
$$

where $\mathbf{x} \in \mathbf{A}_{1}$ and $\mathbf{y} \in \mathbf{A}_{2}$ are the symbols of the joint MCS's with the $\mathbf{A}_{1}, \mathbf{A}_{2}$ alphabets.

The signal $\mathbf{s}$ is transmitted via two independent propagation channels to $\mathrm{UE}_{1}$ and $\mathrm{UE}_{2}$ and the received signal for $k \in 1,2$ user is expressed as

$$
\mathbf{z}_{k}=\alpha_{k} \cdot \mathbf{s}+\mathbf{n}_{k}
$$

where $\mathbf{n}_{k} \sim C N\left(0, N_{0}\right)$ is a component of the complex white Gaussian noise; and $\alpha_{k}$ is a channel propagation coefficient of the $k^{\text {th }}$ user. We denoted above that $\mathrm{UE}_{1}$ possesses a weak propagation 
channel; therefore, $\left|\alpha_{1}\right| \leq\left|\alpha_{2}\right|$. Let us denote $W_{k}$ as SNR of the $k^{\text {th }}$ user. Considering $\mathrm{E}\left\{|\mathbf{s}|^{2}\right\}=1$ (where $\mathrm{E}\{\cdot\}$ - expected value), SNR values relatively received group signal power for two users can be obtained by $W_{k}=\left|\alpha_{k}\right|^{2} / N_{0}$.

The decoding of a PD-NOMA signal is employed by the SIC. Thus, the symbols $\mathbf{x}$ owned by $\mathrm{UE}_{1}$ are decoded firstly using the minimum Euclidean distance criterion and treating $\mathbf{y}$ as an interference. It can be expressed as

$$
\mathbf{x}=\arg \min _{\mathbf{x} \in \mathbf{A}_{1}}\left|\mathbf{z}_{1}-\sqrt{p_{1}} \mathbf{x}\right|^{2}
$$

The symbols $\mathbf{y}$ owned by $\mathrm{UE}_{2}$ are decoded using the SIC by these three steps: first, obtain $\mathbf{x}$ same as $\mathrm{UE}_{1}$ by (3); secondly, cancel $\mathbf{x}$ from the received PD-NOMA signal by $\mathbf{z}_{2}^{*}=\mathbf{z}_{2}-\sqrt{p_{1}} \mathbf{x}$; finally, decode $\mathbf{y}$ from the updated signal $\mathbf{z}_{2}^{*}$.

$$
\mathbf{y}=\arg \min _{\mathbf{y} \in \mathbf{A}_{2}}\left|\mathbf{z}_{2}^{*}-\sqrt{p_{2}} \mathbf{y}\right|^{2}
$$

\section{Proposed joint MCS Table}

In this section, we have described the joint MCS table design process. Our goal is to search for the most efficient joint MCS combination, which guarantees 10\% BLER performance and provides the maximization of the system capacity, considering the user's SNR values. Our approach to the search includes the enumeration of all possible combinations for each joint user's SNR values and their verification with the help of a simulation. The combinations that do not guarantee 10\% BLER are eliminated from a valid range of searches. Then, one with the most SE is selected from the remaining combinations.

\subsection{Used MCS levels}

A downlink system capacity significantly depends on a user non-orthogonal multiplexing technique at the BS side, which includes the joint MCS selection and power allocation between users. Commonly, all possible MCS's are given in a special table in a technical specification. In our system model, we use a bank of the finite number of MCS levels given by [10]. Their main characteristics are presented in Table 1. MCS Table in LTE Downlink.. Each MCS level is indexed by $m$ and based on a square $Q$-QAM and Turbo encoding with the code rate $R$. The SNR threshold with $10 \%$ BLER is used for switching $m$ together with changing the channel state. It allows increasing the SE of transmission due to improving the channel quality.

\subsection{Simulation model}

The structure of the simulation model is shown in Fig. 1. The structure of the simulation model Random bits $\mathbf{b}_{1}, \mathbf{b}_{2}$ are encoded and modulated according to the MCS indices $m_{1}$, $m_{2}$. Next, modulation symbols $\mathbf{x}, \mathbf{y}$ are weighed by the power allocation coefficients $\sqrt{p_{1}}, \sqrt{1-p_{1}}$, and the group PD-NOMA signal $\mathbf{s}$ is obtained by (1). Thereafter, it 
transmits via two AWGN channels by (2) with SNR values $W_{1}, W_{2}$. UE 1 receives $\mathbf{z}_{1}$ and decodes their signal directly by (3) under the channel conditions with the SNR value equal to $W_{1}$; the onward obtains the received bits $\mathbf{b}_{1}$. In their turn, $\mathrm{UE}_{2}$ receives $\mathbf{z}_{2}$ with the SNR value equal to $W_{2}$, then cancels $\mathbf{x}$ by the SIC, and decodes their signal receiving $\mathbf{b}_{2}$ by (4). Next, BLER values for both users are calculated.

Table 1. MCS Table in LTE Downlink.

\begin{tabular}{|c|c|c|c|c|}
\hline$m$ & $Q$ & $\mathrm{R}^{\mathrm{x} 1024}$ & $\mathrm{SE}(\mathrm{bits} / \mathrm{s} / \mathrm{Hz})$ & SNR threshold, dB \\
\hline 1 & 4 & 78 & 0.1523 & -9.478 \\
\hline 2 & 4 & 120 & 0.2344 & -6.658 \\
\hline 3 & 4 & 193 & 0.3770 & -4.098 \\
\hline 4 & 4 & 308 & 0.6010 & -1.798 \\
\hline 5 & 4 & 449 & 0.8770 & 0.399 \\
\hline 6 & 4 & 602 & 1.1758 & 2.424 \\
\hline 7 & 16 & 378 & 1.4766 & 4.489 \\
\hline 8 & 16 & 490 & 1.9141 & 6.367 \\
\hline 9 & 16 & 616 & 2.4063 & 8.456 \\
\hline 10 & 64 & 466 & 2.7305 & 10.266 \\
\hline 11 & 64 & 567 & 3.3223 & 12.218 \\
\hline 12 & 64 & 666 & 3.9023 & 14.122 \\
\hline 13 & 64 & 772 & 4.5234 & 15.849 \\
\hline 14 & 64 & 873 & 5.1152 & 17.786 \\
\hline 15 & 64 & 948 & 5.5547 & 19.809 \\
\hline
\end{tabular}

In the simulation, we change $W_{1}, W_{2}$ from -10 to $40 \mathrm{~dB}$ with $1 \mathrm{~dB}$ step always keeping $W_{1} \leq W_{2}$. For each combination $W_{1}, W_{2}$, we enumerate all possible combinations of MCS levels with the indices $m_{1}, m_{2} \in 1 \ldots 15$ (taken from Table 1. MCS Table in LTE Downlink.) and all possible power allocation coefficients controlled by $p_{1}$ in the range from 0.5 to 1 with the 0.05 step. Next, we initiate the simulation of a data transmission process and estimate a BLER performance. Further, the $m_{1}, m_{2}, p_{1}$ combination that guarantees $10 \%$ BLER performance and maximizes the system capacity is selected and added in the table for the SNR thresholds $W_{1}, W_{2}$. In case the identical sum $\mathrm{SE}$ is given by several combinations, the priority is given to the weak user, and the combination that simultaneously maximizes throughput and the $\mathrm{SE}$ of $\mathrm{UE}_{1}$ is added in the table.

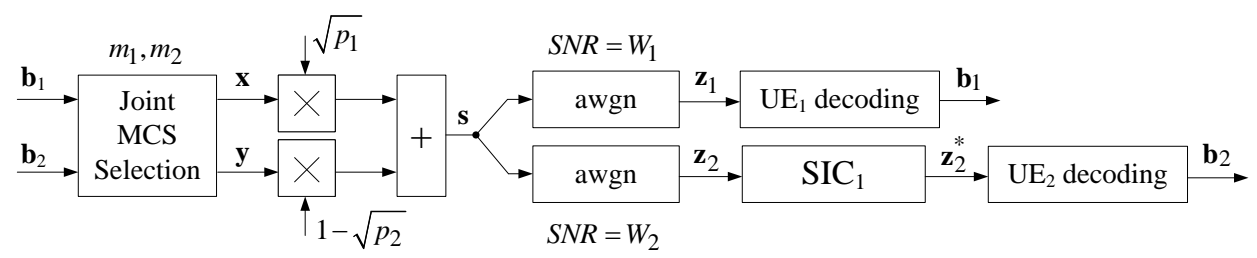

Fig. 1. The structure of the simulation model.

\subsection{Simulation result}


The designed table is given in the Appendix. It consists of 628 various combinations written as $p_{1}\left[m_{1}, m_{2}\right]$ with SNR thresholds $W_{1}, W_{2}$ given in $\mathrm{dB}$. It means that $\mathrm{UE}_{1}$ gets the MCS index $m_{1}$ with the power allocation coefficient $p_{1}$, and $\mathrm{UE}_{2}$ gets $m_{2}$ with $p_{2}=1-p_{1}$. Therefore, the group PD-NOMA signal is obtained by the superposition of user signals by (1) as $\mathbf{s}=\sqrt{p_{1}} \mathbf{x}+\sqrt{1-p_{1}} \mathbf{y}$, where $\mathbf{x} \in \mathbf{A}_{m_{1}}$ and $\mathbf{y} \in \mathbf{A}_{m_{2}}$. For example, $0.950[3,8]$ means that $\mathrm{UE}_{1}$ and $\mathrm{UE}_{2}$ get the $3^{\text {rd }}$ and $9^{\text {th }}$ MCS's from Table 1 . MCS Table in LTE Downlink., and the group PD-NOMA signal is a superposition of the signals using power allocation coefficients $p_{1}=0.95$ and $p_{2}=0.05$, respectively.

There are 32 possible values for $p_{1}$, so only five additional bits are required to transfer the control information about the power shared between the users.

\subsection{Joint MCS's selection}

Exactly as for the AMC in the OMA, in the PD-NOMA, the BS should select the MCS combination with the highest sum SE for the given users' SNR values, considering the thresholds $W_{1}, W_{2}$. These methods are well described in [10]. For example, if $\mathrm{UE}_{1}$ and $\mathrm{UE}_{2}$ possess $S N R_{1}=10 \mathrm{~dB}$ and $S N R_{2}=25 \mathrm{~dB}$, the BS selects from table the combination $0.810[6,14]$ with the SE equal to $6.291 \mathrm{bits} / \mathrm{s} / \mathrm{Hz}$.

There are no joint MCS's that guarantee the required BLER performance within the channel conditions $S N R_{1}<-9 \mathrm{~dB}$ and $S N R_{2}<5 \mathrm{~dB}$. It can be solved with the help of the MCS table (Table 1. MCS Table in LTE Downlink.) extension by adding MCS with a higher BLER performance. Within the conditions $S N R_{1}>38 \mathrm{~dB}$ and $S N R_{2}>39 \mathrm{~dB}$, the BS selects the most efficient MCS's for both users.

\section{Conclusion}

The paper proposes the table of joint MCS's and power allocation coefficients for the downlink two-user PD-NOMA system. The finite number of MCS levels used is taken from the LTE specification and presented in Table 1. MCS Table in LTE Downlink.. The table allows a scheduler to merely pick the MCS for UE1 and UE2 and multiplex them by a power domain based on their SNR values. Hence, the complexity of user PD-NOMA multiplexing by the proposed scheme is well reduced. All multiplexing combinations guarantee $10 \%$ BLER performance at the SNR and satisfy the necessary thresholds. Also, the number of power share states is equal to 32 , and only five redundancy bits are required to transfer it.

Acknowledgments. The work is supported by the Russian Science Foundation grant. Project number 19-79-00087.

\section{References}

1. Y. Saito, A. Benjebbour, Yo. Kishiyama, T. Nakamura, PIMRC, 611 (IEEE, London, 2013)

2. Y. Saito, Y. Kishiyama, A. Benjebbour, T. Nakamura, A. Li, K. Higuchi, VTC, 1 (2013) 
3. Z. Ding, X. Lei, G.K. Karagiannidis, R. Schober, J. Yuan, V.K. Bhargava, IEEE J. on Selected Areas in Commun., 35, 10, 2181 (2017)

4. S.R. Islam, N. Avazov, O.A. Dobre, K.S. Kwak, IEEE Commun. Surveys \& Tutorials, 19, 2, 721 (2016)

5. H. Sadia, M. Zeeshan, S.A. Sheikh, ELEKTRO (IEEE, Zilina, 2018)

6. K. Higuchi, A. Benjebbour, IEICE Transactions on Commun., 98, 3, 403 (2015)

7. A. J. Goldsmith, S. G. Chua, IEEE Trans. Commun., 46, 595 (1998)

8. D. Kim, B. C. Jung, H. Lee, D. K. Sung, H. Yoon, IEEE Trans. Wireless Commun., 7, 12, 5195 (2008)

9. S. Kim, H. Kim, D. Hong, PIMRC (IEEE, Valencia, 2018)

10. J. Fan, Q. Yin, G.Y. Li, B. Peng, X. Zhu, ICCCN (Maui, 2011)

\section{Appendix}

Table 2. Proposed table of the joint MCS's and power allocation coefficients for PD-NOMA system.

\begin{tabular}{|c|c|c|c|c|c|c|c|}
\hline$W_{1}=-9$ & $\overline{W_{2}}$ & $W_{1}=-8$ & $\overline{W_{2}}$ & $W_{1}=-7$ & $\overline{W_{2}}$ & $W_{1}=-6$ & $\overline{W_{2}}$ \\
\hline $0.925[1,1]$ & 5 & $0.865[1,1]$ & 4 & $0.680[1,1]$ & 3 & $0.530[1,1]$ & 2 \\
\hline $0.940[1,2]$ & 7 & $0.805[1,2]$ & 5 & $0.805[1,2]$ & 5 & $0.925[2,1]$ & 5 \\
\hline $0.920[1,3]$ & 8 & $0.875[1,3]$ & 7 & $0.875[1,3]$ & 7 & $0.900[2,2]$ & 6 \\
\hline $0.925[1,4]$ & 10 & $0.835[1,4]$ & 8 & $0.835[1,4]$ & 8 & $0.875[1,3]$ & 7 \\
\hline $0.925[1,5]$ & 12 & $0.870[1,5]$ & 10 & $0.870[1,5]$ & 10 & $0.835[1,4]$ & 8 \\
\hline $0.910[1,6]$ & 13 & $0.830[1,6]$ & 11 & $0.830[1,6]$ & 11 & $0.895[2,4]$ & 9 \\
\hline $0.910[1,7]$ & 15 & $0.785[1,7]$ & 12 & $0.785[1,7]$ & 12 & $0.865[1,5]$ & 10 \\
\hline $0.915[1,8]$ & 17 & $0.815[1,8]$ & 14 & $0.815[1,8]$ & 14 & $0.830[1,6]$ & 11 \\
\hline $0.910[1,9]$ & 19 & $0.820[1,9]$ & 16 & $0.820[1,9]$ & 16 & $0.785[1,7]$ & 12 \\
\hline $0.915[1,10]$ & 21 & $0.770[1,10]$ & 17 & $0.770[1,10]$ & 17 & $0.815[1,8]$ & 14 \\
\hline $0.915[1,11]$ & 23 & $0.780[1,11]$ & 19 & $0.780[1,11]$ & 19 & $0.820[1,9]$ & 16 \\
\hline $0.915[1,12]$ & 25 & $0.790[1,12]$ & 21 & $0.790[1,12]$ & 21 & $0.770[1,10]$ & 17 \\
\hline $0.920[1,13]$ & 27 & $0.805[1,13]$ & 23 & $0.805[1,13]$ & 23 & $0.780[1,11]$ & 19 \\
\hline $0.920[1,14]$ & 29 & $0.810[1,14]$ & 25 & $0.810[1,14]$ & 25 & $0.790[1,12]$ & 21 \\
\hline $0.920[1,15]$ & 31 & $0.750[1,15]$ & 26 & $0.750[1,15]$ & 26 & $0.805[1,13]$ & 23 \\
\hline$W_{1}=-5$ & $W_{2}$ & $W_{1}=-4$ & $W_{2}$ & $W_{1}=-3$ & $W_{2}$ & $0.805[1,14]$ & 25 \\
\hline $0.530[1,1]$ & 2 & $0.530[1,1]$ & 2 & $0.530[1,1]$ & 2 & $0.750[1,15]$ & 26 \\
\hline $0.860[2,1]$ & 4 & $0.675[2,1]$ & 3 & $0.680[2,1]$ & 3 & $0.900[2,15]$ & 30 \\
\hline $0.805[2,2]$ & 5 & $0.805[2,2]$ & 5 & $0.860[3,1]$ & 4 & $W_{1}=-2$ & $W_{2}$ \\
\hline $0.875[2,3]$ & 7 & $0.875[2,3]$ & 7 & $0.900[3,2]$ & 6 & $0.530[2,1]$ & 2 \\
\hline $0.835[2,4]$ & 8 & $0.835[2,4]$ & 8 & $0.875[3,3]$ & 7 & $0.865[3,1]$ & 4 \\
\hline $0.870[2,5]$ & 10 & $0.870[2,5]$ & 10 & $0.835[2,4]$ & 8 & $0.805[3,2]$ & 5 \\
\hline $0.830[2,6]$ & 11 & $0.830[2,6]$ & 11 & $0.895[3,4]$ & 9 & $0.870[3,3]$ & 7 \\
\hline $0.785[2,7]$ & 12 & $0.785[2,7]$ & 12 & $0.870[3,5]$ & 10 & $0.830[3,4]$ & 8 \\
\hline $0.815[2,8]$ & 14 & $0.815[2,8]$ & 14 & $0.830[2,6]$ & 11 & $0.870[3,5]$ & 10 \\
\hline $0.820[2,9]$ & 16 & $0.820[2,9]$ & 16 & $0.785[2,7]$ & 12 & $0.830[3,6]$ & 11 \\
\hline $0.770[2,10]$ & 17 & $0.770[2,10]$ & 17 & $0.850[3,7]$ & 13 & $0.790[3,7]$ & 12 \\
\hline $0.785[2,11]$ & 19 & $0.780[2,11]$ & 19 & $0.815[2,8]$ & 14 & $0.815[3,8]$ & 14 \\
\hline $0.790[2,12]$ & 21 & $0.790[2,12]$ & 21 & $0.860[3,8]$ & 15 & $0.820[3,9]$ & 16 \\
\hline $0.805[2,13]$ & 23 & $0.805[2,13]$ & 23 & $0.820[2,9]$ & 16 & $0.770[3,10]$ & 17 \\
\hline $0.805[2,14]$ & 25 & $0.805[2,14]$ & 25 & $0.770[2,10]$ & 17 & $0.780[3,11]$ & 19 \\
\hline $0.750[1,15]$ & 26 & $0.750[2,15]$ & 26 & $0.780[2,11]$ & 19 & $0.790[3,12]$ & 21 \\
\hline $0.805[2,15]$ & 27 & $0.985[3,15]$ & 39 & $0.790[2,12]$ & 21 & $0.805[3,13]$ & 23 \\
\hline
\end{tabular}




\begin{tabular}{|c|c|c|c|c|c|c|c|}
\hline$W_{1}=-1$ & $W_{2}$ & $0.765[2,15]$ & 26 & $0.805[2,13]$ & 23 & $0.805[3,14]$ & 25 \\
\hline $0.530[2,1]$ & 2 & $0.985[3,15]$ & 40 & $0.845[3,13]$ & 24 & $0.750[3,15]$ & 26 \\
\hline $0.680[3,1]$ & 3 & $W_{1}=0$ & $W_{2}$ & $0.805[2,14]$ & 25 & $W_{1}=2$ & $W_{2}$ \\
\hline $0.925[4,1]$ & 5 & $0.530[2,1]$ & 2 & $0.750[2,15]$ & 26 & $0.535[3,1]$ & 2 \\
\hline $0.940[4,2]$ & 7 & $0.675[3,1]$ & 3 & $0.845[3,15]$ & 28 & $0.680[4,1]$ & 3 \\
\hline $0.920[4,3]$ & 8 & $0.860[4,1]$ & 4 & $W_{1}=1$ & $W_{2}$ & $0.865[5,1]$ & 4 \\
\hline $0.870[3,5]$ & 10 & $0.805[4,2]$ & 5 & $0.530[2,1]$ & 2 & $0.900[5,2]$ & 6 \\
\hline $0.830[3,6]$ & 11 & $0.875[4,3]$ & 7 & $0.685[3,1]$ & 3 & $0.875[5,3]$ & 7 \\
\hline $0.785[3,7]$ & 12 & $0.835[4,4]$ & 8 & $0.860[4,1]$ & 4 & $0.895[5,4]$ & 9 \\
\hline $0.815[3,8]$ & 14 & $0.870[4,5]$ & 10 & $0.805[4,2]$ & 5 & $0.870[5,5]$ & 10 \\
\hline $0.820[3,9]$ & 16 & $0.830[4,6]$ & 11 & $0.950[5,1]$ & 6 & $0.830[4,6]$ & 11 \\
\hline $0.770[3,10]$ & 17 & $0.785[3,7]$ & 12 & $0.940[5,2]$ & 7 & $0.785[4,7]$ & 12 \\
\hline $0.785[3,11]$ & 19 & $0.850[4,7]$ & 13 & $0.835[4,4]$ & 8 & $0.815[4,8]$ & 14 \\
\hline $0.790[3,12]$ & 21 & $0.815[4,8]$ & 14 & $0.940[5,3]$ & 9 & $0.860[5,8]$ & 15 \\
\hline $0.805[3,13]$ & 23 & $0.820[4,9]$ & 16 & $0.870[4,5]$ & 10 & $0.820[4,9]$ & 16 \\
\hline $0.805[3,14]$ & 25 & $0.770[3,10]$ & 17 & $0.830[4,6]$ & 11 & $0.770[4,10]$ & 17 \\
\hline $0.750[2,15]$ & 26 & $0.830[4,10]$ & 18 & $0.785[4,7]$ & 12 & $0.780[4,11]$ & 19 \\
\hline $0.805[3,15]$ & 27 & $0.785[3,11]$ & 19 & $0.815[4,8]$ & 14 & $0.790[4,12]$ & 21 \\
\hline $0.920[4,15]$ & 31 & $0.830[4,11]$ & 20 & $0.820[4,9]$ & 16 & $0.805[4,13]$ & 23 \\
\hline$W_{1}=3$ & $W_{2}$ & $0.790[3,12]$ & 21 & $0.770[4,10]$ & 17 & $0.805[4,14]$ & 25 \\
\hline $0.675[4,1]$ & 3 & $0.835[4,12]$ & 22 & $0.785[4,11]$ & 19 & $0.750[4,15]$ & 26 \\
\hline $0.865[5,1]$ & 4 & $0.805[4,13]$ & 23 & $0.790[4,12]$ & 21 & $0.875[5,15]$ & 29 \\
\hline $0.805[5,2]$ & 5 & $0.810[4,14]$ & 25 & $0.805[4,13]$ & 23 & $W_{1}=6$ & $W_{2}$ \\
\hline $0.965[6,1]$ & 7 & $0.750[3,15]$ & 26 & $0.805[4,14]$ & 25 & $0.900[6,2]$ & 6 \\
\hline $0.835[5,4]$ & 8 & $0.805[4,15]$ & 27 & $0.750[3,15]$ & 26 & $0.875[6,3]$ & 7 \\
\hline $0.870[5,5]$ & 10 & $W_{1}=4$ & $W_{2}$ & $0.805[4,15]$ & 27 & $0.830[6,4]$ & 8 \\
\hline $0.830[5,6]$ & 11 & $0.865[5,1]$ & 4 & $0.950[5,15]$ & 33 & $0.870[6,5]$ & 10 \\
\hline $0.785[4,7]$ & 12 & $0.925[6,1]$ & 5 & $W_{1}=5$ & $W_{2}$ & $0.830[6,6]$ & 11 \\
\hline $0.850[5,7]$ & 13 & $0.900[6,2]$ & 6 & $0.925[6,1]$ & 5 & $0.785[5,7]$ & 12 \\
\hline $0.815[5,8]$ & 14 & $0.920[6,3]$ & 8 & $0.900[6,2]$ & 6 & $0.850[6,7]$ & 13 \\
\hline $0.820[5,9]$ & 16 & $0.895[6,4]$ & 9 & $0.875[6,3]$ & 7 & $0.815[6,8]$ & 14 \\
\hline $0.770[4,10]$ & 17 & $0.900[6,5]$ & 11 & $0.895[6,4]$ & 9 & $0.820[6,9]$ & 16 \\
\hline $0.830[5,10]$ & 18 & $0.785[5,7]$ & 12 & $0.870[6,5]$ & 10 & $0.770[5,10]$ & 17 \\
\hline $0.780[4,11]$ & 19 & $0.815[5,8]$ & 14 & $0.785[5,7]$ & 12 & $0.830[6,10]$ & 18 \\
\hline $0.830[5,11]$ & 20 & $0.820[5,9]$ & 16 & $0.850[6,7]$ & 13 & $0.780[5,11]$ & 19 \\
\hline $0.790[4,12]$ & 21 & $0.770[5,10]$ & 17 & $0.815[5,8]$ & 14 & $0.830[6,11]$ & 20 \\
\hline $0.835[5,12]$ & 22 & $0.780[5,11]$ & 19 & $0.860[6,8]$ & 15 & $0.790[5,12]$ & 21 \\
\hline $0.805[5,13]$ & 23 & $0.790[5,12]$ & 21 & $0.820[5,9]$ & 16 & $0.835[6,12]$ & 22 \\
\hline $0.810[5,14]$ & 25 & $0.805[5,13]$ & 23 & $0.770[5,10]$ & 17 & $0.805[6,13]$ & 23 \\
\hline $0.750[4,15]$ & 26 & $0.805[5,14]$ & 25 & $0.780[5,11]$ & 19 & $0.805[6,14]$ & 25 \\
\hline $0.805[5,15]$ & 27 & $0.750[5,15]$ & 26 & $0.790[5,12]$ & 21 & $0.805[6,15]$ & 27 \\
\hline $0.950[6,15]$ & 34 & $0.900[6,15]$ & 30 & $0.805[5,13]$ & 23 & $0.950[7,15]$ & 33 \\
\hline$W_{1}=7$ & $W_{2}$ & $W_{1}=8$ & $W_{2}$ & $0.845[6,13]$ & 24 & $W_{1}=10$ & $W_{2}$ \\
\hline $0.875[6,6]$ & 7 & $0.835[6,4]$ & 8 & $0.810[5,14]$ & 25 & $0.870[6,5]$ & 10 \\
\hline $0.835[6,4]$ & 8 & $0.870[6,5]$ & 10 & $0.750[5,15]$ & 26 & $0.830[6,6]$ & 11 \\
\hline $0.870[6,5]$ & 10 & $0.830[6,6]$ & 11 & $0.845[6,15]$ & 28 & $0.785[6,7]$ & 12 \\
\hline $0.830[6,6]$ & 11 & $0.785[6,7]$ & 12 & $0.985[7,15]$ & 39 & $0.815[6,8]$ & 14 \\
\hline $0.785[6,7]$ & 12 & $0.815[6,8]$ & 14 & $W_{1}=9$ & $W_{2}$ & $0.820[6,9]$ & 16 \\
\hline $0.815[6,8]$ & 14 & $0.820[6,9]$ & 16 & $0.895[6,4]$ & 9 & $0.770[6,10]$ & 17 \\
\hline $0.820[6,9]$ & 16 & $0.770[6,10]$ & 17 & $0.870[6,5]$ & 10 & $0.785[6,11]$ & 19 \\
\hline $0.770[5,10]$ & 17 & $0.780[6,11]$ & 19 & $0.830[6,6]$ & 11 & $0.790[6,12]$ & 21 \\
\hline $0.830[6,10]$ & 18 & $0.790[6,12]$ & 21 & $0.785[6,7]$ & 12 & $0.805[6,13]$ & 23 \\
\hline
\end{tabular}




\begin{tabular}{|c|c|c|c|c|c|c|c|}
\hline $0.785[6,11]$ & 19 & $0.805[6,13]$ & 23 & $0.815[6,8]$ & 14 & $0.810[6,14]$ & 25 \\
\hline $0.790[6,12]$ & 21 & $0.805[6,14]$ & 25 & $0.820[6,9]$ & 16 & $0.750[6,15]$ & 26 \\
\hline $0.805[6,13]$ & 23 & $0.750[5,15]$ & 26 & $0.770[6,10]$ & 17 & $0.950[8,14]$ & 31 \\
\hline $0.805[6,14]$ & 25 & $0.805[6,15]$ & 27 & $0.780[6,11]$ & 19 & $0.965[9,14]$ & 33 \\
\hline $0.750[5,15]$ & 26 & $0.950[8,14]$ & 31 & $0.790[6,12]$ & 21 & $0.965[9,15]$ & 35 \\
\hline $0.805[6,15]$ & 27 & $0.950[8,15]$ & 33 & $0.805[6,13]$ & 23 & $W_{1}=14$ & $W_{2}$ \\
\hline $0.950[7,15]$ & 33 & $W_{1}=12$ & $\overline{W_{2}}$ & $0.805[6,14]$ & 25 & $0.815[6,8]$ & 14 \\
\hline $0.965[8,15]$ & 36 & $0.785[6,7]$ & 12 & $0.750[6,15]$ & 26 & $0.820[6,9]$ & 16 \\
\hline$W_{1}=11$ & $\overline{W_{2}}$ & $0.815[6,8]$ & 14 & $0.950[8,14]$ & 31 & $0.770[6,10]$ & 17 \\
\hline $0.830[6,6]$ & 11 & $0.820[6,9]$ & 16 & $0.950[8,15]$ & 33 & $0.780[6,11]$ & 19 \\
\hline $0.785[6,7]$ & 12 & $0.770[6,10]$ & 17 & $0.990[9,14]$ & 38 & $0.790[6,12]$ & 21 \\
\hline $0.815[6,8]$ & 14 & $0.780[6,11]$ & 19 & $0.990[9,15]$ & 40 & $0.805[6,13]$ & 23 \\
\hline $0.820[6,9]$ & 16 & $0.790[6,12]$ & 21 & $W_{1}=13$ & $W_{2}$ & $0.805[6,14]$ & 25 \\
\hline $0.770[6,10]$ & 17 & $0.805[6,13]$ & 23 & $0.850[6,7]$ & 13 & $0.750[6,15]$ & 26 \\
\hline $0.780[6,11]$ & 19 & $0.805[6,14]$ & 25 & $0.815[6,8]$ & 14 & $0.950[9,13]$ & 29 \\
\hline $0.790[6,12]$ & 21 & $0.750[6,15]$ & 26 & $0.820[6,9]$ & 16 & $0.950[9,14]$ & 31 \\
\hline $0.805[6,13]$ & 23 & $0.950[9,13]$ & 29 & $0.770[6,10]$ & 17 & $0.950[9,15]$ & 33 \\
\hline $0.805[6,14]$ & 25 & $0.950[9,14]$ & 31 & $0.780[6,11]$ & 19 & $0.985[11,14]$ & 37 \\
\hline $0.750[6,15]$ & 26 & $0.950[9,15]$ & 33 & $0.790[6,12]$ & 21 & $0.985[11,15]$ & 39 \\
\hline $0.950[9,13]$ & 29 & $0.985[10,15]$ & 39 & $0.805[6,13]$ & 23 & $W_{1}=18$ & $\overline{W_{2}}$ \\
\hline $0.950[9,14]$ & 31 & $W_{1}=16$ & $\overline{W_{2}}$ & $0.805[6,14]$ & 25 & $0.990[12,1]$ & 18 \\
\hline $0.950[9,15]$ & 33 & $0.820[6,9]$ & 16 & $0.750[6,15]$ & 26 & $0.995[13,1]$ & 19 \\
\hline $0.990[10,15]$ & 40 & $0.770[6,10]$ & 17 & $0.950[9,13]$ & 29 & $0.995[13,2]$ & 20 \\
\hline$W_{1}=15$ & $\overline{W_{2}}$ & $0.990[12,1]$ & 18 & $0.950[9,14]$ & 31 & $0.790[6,12]$ & 21 \\
\hline $0.860[6,8]$ & 15 & $0.780[6,11]$ & 19 & $0.950[9,15]$ & 33 & $0.805[6,13]$ & 23 \\
\hline $0.820[6,9]$ & 16 & $0.790[6,12]$ & 21 & $0.985[10,15]$ & 39 & $0.805[6,14]$ & 25 \\
\hline $0.770[6,10]$ & 17 & $0.805[6,13]$ & 23 & $W_{1}=17$ & $W_{2}$ & $0.750[6,15]$ & 26 \\
\hline $0.780[6,11]$ & 19 & $0.805[6,14]$ & 25 & $0.770[6,10]$ & 17 & $0.950[9,13]$ & 29 \\
\hline $0.790[6,12]$ & 21 & $0.750[6,15]$ & 26 & $0.990[12,1]$ & 18 & $0.950[9,14]$ & 31 \\
\hline $0.805[6,13]$ & 23 & $0.950[9,13]$ & 29 & $0.995[13,1]$ & 19 & $0.950[9,15]$ & 33 \\
\hline $0.805[6,14]$ & 25 & $0.950[9,14]$ & 31 & $0.995[13,2]$ & 20 & $0.985[12,13]$ & 35 \\
\hline $0.750[6,15]$ & 26 & $0.950[9,15]$ & 33 & $0.790[6,12]$ & 21 & $0.985[12,14]$ & 37 \\
\hline $0.950[9,13]$ & 29 & $0.990[12,13]$ & 36 & $0.805[6,13]$ & 23 & $0.985[12,15]$ & 39 \\
\hline $0.950[9,14]$ & 31 & $0.985[11,14]$ & 37 & $0.805[6,14]$ & 25 & $W_{1}=22$ & $W_{2}$ \\
\hline $0.950[9,15]$ & 33 & $0.990[12,14]$ & 38 & $0.750[5,15]$ & 26 & $0.995[15,3]$ & 22 \\
\hline $0.985[11,14]$ & 37 & $0.990[12,15]$ & 40 & $0.805[6,15]$ & 27 & $0.995[15,4]$ & 23 \\
\hline $0.985[11,15]$ & 39 & $W_{1}=20$ & $\overline{W_{2}}$ & $0.950[9,13]$ & 29 & $0.995[15,5]$ & 24 \\
\hline$W_{1}=19$ & $\overline{W_{2}}$ & $0.995[14,2]$ & 20 & $0.950[9,14]$ & 31 & $0.995[15,6]$ & 26 \\
\hline $0.990[13,2]$ & 19 & $0.995[14,3]$ & 21 & $0.950[9,15]$ & 33 & $0.990[14,8]$ & 27 \\
\hline $0.990[13,3]$ & 20 & $0.995[14,4]$ & 23 & $0.985[12,13]$ & 35 & $0.990[14,9]$ & 29 \\
\hline $0.790[6,12]$ & 21 & $0.995[14,5]$ & 24 & $0.985[12,14]$ & 37 & $0.990[14,10]$ & 31 \\
\hline $0.990[13,4]$ & 22 & $0.810[6,14]$ & 25 & $0.985[12,15]$ & 39 & $0.990[14,11]$ & 33 \\
\hline $0.805[6,13]$ & 23 & $0.750[6,15]$ & 26 & $W_{1}=21$ & $W_{2}$ & $0.985[13,13]$ & 35 \\
\hline $0.805[6,14]$ & 25 & $0.990[13,9]$ & 29 & $0.995[14,3]$ & 21 & $0.990[14,13]$ & 36 \\
\hline $0.750[6,15]$ & 26 & $0.995[14,8]$ & 30 & $0.995[14,4]$ & 23 & $0.990[14,14]$ & 38 \\
\hline $0.990[13,9]$ & 29 & $0.950[9,14]$ & 31 & $0.995[14,5]$ & 24 & $0.990[14,15]$ & 40 \\
\hline $0.950[9,14]$ & 31 & $0.950[9,15]$ & 33 & $0.805[6,14]$ & 25 & $W_{1}=26$ & $W_{2}$ \\
\hline $0.950[9,15]$ & 33 & $0.990[13,12]$ & 35 & $0.750[6,15]$ & 26 & $0.990[15,7]$ & 26 \\
\hline $0.990[13,12]$ & 35 & $0.990[13,13]$ & 36 & $0.985[13,9]$ & 28 & $0.990[15,8]$ & 27 \\
\hline $0.990[13,13]$ & 36 & $0.990[13,14]$ & 38 & $0.985[13,10]$ & 30 & $0.985[14,9]$ & 28 \\
\hline $0.990[13,14]$ & 38 & $0.990[13,15]$ & 40 & $0.950[9,14]$ & 31 & $0.990[15,9]$ & 29 \\
\hline$W_{1}=23$ & $\overline{W_{2}}$ & $W_{1}=24$ & $\overline{W_{2}}$ & $0.985[13,11]$ & 32 & $0.990[15,10]$ & 31 \\
\hline
\end{tabular}




\begin{tabular}{|c|c|c|c|c|c|c|c|}
\hline $0.995[15,4]$ & 23 & $0.990[15,6]$ & 24 & $0.985[13,12]$ & 33 & $0.985[14,11]$ & 32 \\
\hline $0.995[15,5]$ & 24 & $0.990[14,8]$ & 27 & $0.985[13,13]$ & 35 & $0.985[14,12]$ & 33 \\
\hline $0.995[15,6]$ & 26 & $0.995[15,7]$ & 28 & $0.985[13,14]$ & 37 & $0.985[14,13]$ & 35 \\
\hline $0.990[14,8]$ & 27 & $0.990[14,9]$ & 29 & $0.985[13,15]$ & 39 & $0.990[15,13]$ & 36 \\
\hline $0.995[15,7]$ & 28 & $0.990[14,10]$ & 31 & $W_{1}=25$ & $W_{2}$ & $0.985[14,14]$ & 37 \\
\hline $0.990[14,9]$ & 29 & $0.995[15,9]$ & 32 & $0.990[15,6]$ & 25 & $0.990[15,14]$ & 38 \\
\hline $0.990[14,10]$ & 31 & $0.990[14,11]$ & 33 & $0.990[14,8]$ & 27 & $0.990[15,15]$ & 40 \\
\hline $0.995[15,9]$ & 32 & $0.985[13,13]$ & 35 & $0.985[14,9]$ & 28 & $W_{1}=30$ & $W_{2}$ \\
\hline $0.990[14,11]$ & 33 & $0.990[14,13]$ & 36 & $0.990[14,10]$ & 31 & $0.990[15,9]$ & 30 \\
\hline $0.985[13,13]$ & 35 & $0.990[14,14]$ & 38 & $0.995[15,9]$ & 32 & $0.990[15,10]$ & 31 \\
\hline $0.990[14,13]$ & 36 & $0.990[14,15]$ & 40 & $0.990[14,11]$ & 33 & $0.985[14,11]$ & 32 \\
\hline $0.990[14,14]$ & 38 & $W_{1}=28$ & $W_{2}$ & $0.985[13,13]$ & 35 & $0.985[14,12]$ & 33 \\
\hline $0.990[14,15]$ & 40 & $0.985[14,9]$ & 28 & $0.990[14,13]$ & 36 & $0.985[14,13]$ & 35 \\
\hline$W_{1}=27$ & $\overline{W_{2}}$ & $0.990[15,9]$ & 29 & $0.990[14,14]$ & 38 & $0.990[15,13]$ & 36 \\
\hline $0.990[15,8]$ & 27 & $0.990[15,10]$ & 31 & $0.990[14,15]$ & 40 & $0.985[14,14]$ & 37 \\
\hline $0.985[14,9]$ & 28 & $0.985[14,11]$ & 32 & $W_{1}=29$ & $W_{2}$ & $0.990[15,14]$ & 38 \\
\hline $0.990[15,9]$ & 29 & $0.985[14,12]$ & 33 & $0.990[15,9]$ & 29 & $0.990[15,15]$ & 40 \\
\hline $0.990[15,10]$ & 31 & $0.985[14,13]$ & 35 & $0.990[15,10]$ & 31 & $W_{1}=34$ & $\overline{W_{2}}$ \\
\hline $0.985[14,11]$ & 32 & $0.990[15,13]$ & 36 & $0.985[14,11]$ & 32 & $0.985[15,12]$ & 34 \\
\hline $0.985[14,12]$ & 33 & $0.985[14,14]$ & 37 & $0.985[14,12]$ & 33 & $0.985[15,13]$ & 35 \\
\hline $0.985[14,13]$ & 35 & $0.990[15,14]$ & 38 & $0.985[14,13]$ & 35 & $0.985[15,14]$ & 37 \\
\hline $0.985[14,14]$ & 37 & $0.990[15,15]$ & 40 & $0.990[15,13]$ & 36 & $0.985[15,15]$ & 39 \\
\hline $0.990[15,14]$ & 38 & $W_{1}=32$ & $W_{2}$ & $0.985[14,14]$ & 37 & $W_{1}=36$ & $W_{2}$ \\
\hline $0.990[15,15]$ & 40 & $0.985[15,11]$ & 32 & $0.990[15,14]$ & 38 & $0.990[15,13]$ & 36 \\
\hline$W_{1}=31$ & $W_{2}$ & $0.985[15,12]$ & 33 & $0.990[15,15]$ & 40 & $0.985[15,14]$ & 37 \\
\hline $0.990[15,10]$ & 31 & $0.985[15,13]$ & 35 & $W_{1}=33$ & $W_{2}$ & $0.985[15,15]$ & 39 \\
\hline $0.985[14,11]$ & 32 & $0.985[15,14]$ & 37 & $0.985[15,12]$ & 33 & $W_{1}=37$ & $W_{2}$ \\
\hline $0.985[14,12]$ & 33 & $0.985[15,15]$ & 39 & $0.985[15,13]$ & 35 & $0.985[15,14]$ & 37 \\
\hline $0.985[14,13]$ & 35 & $W_{1}=35$ & $W_{2}$ & $0.985[15,14]$ & 37 & $0.985[15,15]$ & 39 \\
\hline $0.990[15,13]$ & 36 & $0.985[15,13]$ & 35 & $0.985[15,15]$ & 39 & $W_{1}=38$ & $\overline{W_{2}}$ \\
\hline $0.985[14,14]$ & 37 & $0.985[15,14]$ & 37 & & $0.990[15,14]$ & 38 \\
\hline $0.990[15,14]$ & 38 & $0.985[15,15]$ & 39 & & & $0.985[15,15]$ & 39 \\
\hline $0.990[15,15]$ & 40 & & & & & & \\
\hline
\end{tabular}

